\title{
Calprotectin can play an inflammatory role in acne vulgaris
}

\author{
Selma Korkmaz ${ }^{1}$, Sezin Kuru Fıçıcıoğlu² \\ ${ }^{1}$ Faculty of Medicine, Süleyman Demirel University, Isparta, Turkey \\ ${ }^{2}$ Faculty of Medicine, Trakya University, Edirne, Turkey
}

\author{
Adv Dermatol Allergol 2018; XXXV (4): 397-399 \\ DOI: https://doi.org/10.5114/ada.2017.71286
}

\begin{abstract}
Introduction: Acne vulgaris (AV) is a chronic inflammatory disorder of the pilosebaceous unit. Although various mechanisms have been indicated in the etiopathogenesis of acne vulgaris, the exact pathophysiology is still unknown. Aim: To investigate the level of calprotectin in acne vulgaris and its levels relationship with disease severity. Material and methods: A total of 66 AV patients, who were divided into 33 mild and 33 moderate-severe cases, and 30 healthy controls were enrolled in the study. Disease severity was assessed using the Global Acne Score. According to this scale, patients whose Global Acne Score was 1-18 had mild acne, those with a score of 19-30 had moderate acne, those with a score of 31-38 had severe acne, and those with a score greater than 39 had very severe acne. Serum calprotectin levels of all participants were measured by enzyme-linked immunosorbent assay method. Results: The serum calprotectin levels in the moderate-severe AV group were significantly higher than that of the mild AV group ( $p$ 0.001). In addition, the serum calprotectin level in the mild AV group was significantly higher than that of the healthy control group $(p=0.047)$. However, in the Spearman's correlation analysis, the serum calprotectin level and GAS were not correlated in AV patients $(p=0.171, r=0.179)$.

Conclusions: Serum calprotectin levels are increased in mild and moderate AV patients.
\end{abstract}

Key words: acne vulgaris, calprotectin, inflammation.

\section{Introduction}

Acne vulgaris (AV) is an inflammatory skin disease of the pilosebaceous unit. There are numerous factors in the etiopathogenesis of AV. The main factors are inflammation, abnormal keratinization, microbial flora changes, and increased sebum production. The sequence in which these events occur is still not certain, but recently, inflammation has been suggested as the initial factor. The mechanisms behind the beginning and maintenance of the inflammatory response are not fully known, but Propionibacterium acnes plays an important role in these mechanisms [1-3]. Propionibacterium acnes triggers inflammation both directly and indirectly; it increases proinflammatory cytokine release and antimicrobial peptide expression. Propionibacterium acnes also stimulates the innate immune response by activating Toll-like receptor (TLR)-2. Sebocytes are the major cells within sebaceous glands that constitute the pilosebaceous unit along with hair, hair follicles, and the arrector pili muscles. Along with the keratinocytes, sebocytes act as immune cells [4-6].
Calprotectin is the heterodimer form of S100A8 and S100A9, which are members of the S100 protein family and which play a role in various inflammatory process. Previously, calprotectin was also called calgranulin (due to its calcium binding property and because it is mostly found in granulocytes), leukocyte-derived protein, cystic fibrosis antigen, and migration inhibitory factor-related proteins 8 and 14 . High amounts of calprotectin are found in neutrophils, and calprotectin is released from activated neutrophils and monocytes. Other cells, such as osteoclasts, chondrocytes, keratinocytes, endothelial cells and fibroblast-like synoviocytes, can also secrete calprotectin. Calprotectin plays a role in infections, leukocyte migration, and cytoskeleton regulation. In addition, calprotectin binds to $\mathrm{Zn}^{2+}$ and $\mathrm{Mn}^{2+}$, which are metal nutrients for bacteria, and this binding produces a direct antimicrobial effect.

Calprotectin can be detected in many body fluids, including feces, serum, saliva, and synovial fluid. Serum calprotectin levels increase in various cancers, inflammatory diseases and autoimmune diseases [7-12]. In addi-

Address for correspondence: Selma Korkmaz, Faculty of Medicine, Süleyman Demirel University, 32040 Isparta, Turkey, phone: +90 5063567227, e-mail: selmakorkmaz35@gmail.com Received: 28.05.2017, accepted: 7.07.2017. 
tion, calprotectin has been found to be more accurate than other laboratory markers of inflammation, such as C-reactive protein (CRP) and erythrocyte sedimentation rate (ESR). Since calprotectin can be detected with minimal inflammation and because it normalizes prior to CRP and ESR, calprotectin can be used to follow up with disease activity through the evaluation of treatment response or disease relapse, especially with autoimmune disorders $[13,14]$.

\section{Aim}

However, to our knowledge, no research has evaluated the role of calprotectin in AV patients. In this study, we aimed to investigate the level and importance of calprotectin in AV.

\section{Material and methods}

The study was initiated upon obtaining approval from the ethics committee of the Trakya University Medical Faculty. Informed consent was obtained from all subjects before the study began.

\section{Patient groups and study protocol}

This prospective study was conducted in the Department of Dermatology, Medical Faculty, Trakya University. A total of 66 patients with acne vulgaris (37 females, 29 males, mean age: $20.84 \pm 1.98$ ) and 30 healthy controls (17 females, 13 males, mean age: $23.37 \pm 33.82$ ) were enrolled in the study. We evaluated the acne severity of the patients using the Global Acne Grading System [15]. According to this scale, patients whose Global Acne Score (GAS) was 1-18 have mild acne, those with a score of 19-30 have moderate acne, those with a score of 31-38 have severe acne, and those with a score greater than 39 have very severe acne. We divided our acne patients

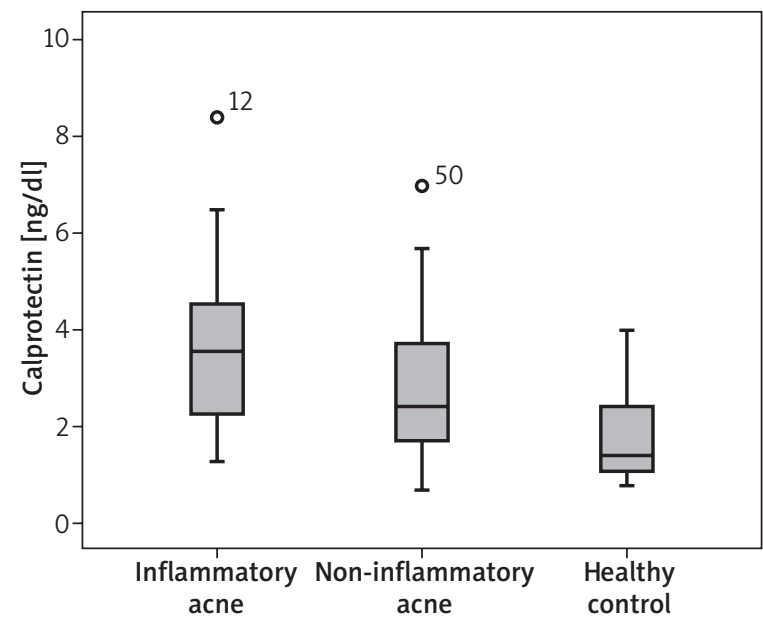

Figure 1. The calprotectin levels of all groups into two groups: mild acne (GAS 1-18) and moderate-severe acne (GAS 18-39). During the first assessment, a detailed medical history was obtained, and a physical examination was performed by an experienced dermatologist.

Subjects with a systemic disease (diabetes mellitus, heart failure, cirrhosis, infection, renal failure, collagen disease, pregnancy, or malignancy), with endocrinopathies (such as Cushing's syndrome, Addison's syndrome, polycystic ovary syndrome, pheochromocytoma, or hyper- and hypothyroidism) or who had been using nonsteroid anti-inflammatory drugs or hormonal medicines, such as steroids, were excluded from the study.

We took a 10-cc venous blood sample from each included patient, centrifuged the sample at 3000 rpm for $5 \mathrm{~min}$, and measured the serum calprotectin level of each sample using the enzyme-linked immunosorbent assay (ELISA) method (Cusabio).

\section{Statistical analysis}

Statistical analyses were performed using SPSS version 22.0 program and $p<0.05$ was accepted as statistically significant.

\section{Results}

The patient groups were similar according to their median age and gender ( $p>0.05$ for each parameter). In the mild acne group, the median GAS was $14.23 \pm 4.86$, and in the moderate-severe acne group, the GAS was $29.30 \pm 2.46$, which was significantly higher than that of the mild group $(p<0.001)$.

The serum calprotectin level of the moderate-severe AV group was significantly higher than that of the mild AV group ( $p<0.001)$. In addition, the serum calprotectin level of the mild AV group was significantly higher than that of the healthy control group $(p=0.047)$.

For the AV patients, there was no correlation between serum calprotectin level and GAS, as determined using Spearman's correlation analysis ( $p=0.171, r=0.179)$ (Figure 1).

\section{Discussion}

In this study, the serum calprotectin level was increased in AV patients, and this increase was more prevalent in moderate and severe cases of AV. These results indicate that the serum calprotectin level is increased in accordance with inflammation and that calprotectin can have a role in the inflammation that occurs with AV.

Calprotectin is released from keratinocytes along with activated monocytes, phagocytes, granulocytes and vascular cells. Calprotectin is recognized by TLRs and induces inflammation [11, 12], but calprotectin has not been evaluated in AV patients before. In this study, the serum calprotectin level of patients with AV was statistically significantly higher than that of the healthy controls. In addition, the serum calprotectin level was higher in 
moderate-severe AV patients than in mild AV patients. According to these results, a high calprotectin level might play a role in the inflammation process of $\mathrm{AV}$.

The role of the microorganisms colonizing the pilosebaceous unit during the inflammatory response of AV is already known. The most important of these microorganisms is $P$. acnes. Sebocytes are the major components of the pilosebaceous unit. They act as immune cells and express TLR-2, TLR-4, CD1d, and CD14, which are molecules of the innate immune system. Propionibacterium acnes activates keratinocytes and sebocytes through the intervention of TLRS, CD14, and CD1. This interaction means that inflammation exists from the beginning of the microcomedone stage [16].

Fecal calprotectin has been used successfully to monitor inflammatory bowel disease and other gastrointestinal diseases, like necrotizing enterocolitis [17]. Calprotectin levels are also increased in rheumatic diseases, like psoriatic arthritis, rheumatoid arthritis and juvenile idiopathic arthritis. The serum calprotectin level has been used to diagnose neonatal sepsis [8, 10, 18, 19]. In multiple sclerosis cases, calprotectin can be used to determine disease severity and neuroinflammation [14]. Calprotectin can be released from the epithelium and increase the proinflammatory response, and calprotectin can also protect against inflammation-induced skin carcinogenesis [20].

Increased calprotectin levels have been found in various inflammatory diseases, so calprotectin might be useful for the diagnosis and determination of disease severity. Calprotectin is stable and easily measurable in various body fluids, but calprotectin levels can be detected with either commercial kits or homemade ELISA tests, each of which has different sensitivities and cutoff levels. Therefore, a standardized method to measure the calprotectin level is required.

Gaining knowledge about AV's inflammation and related mechanisms can lead to new treatment modalities. In our study, the increased serum calprotectin levels in AV patients suggested that calprotectin can be used to determine disease severity and evaluate a patient's response to treatment. Calprotectin might also be a possible target in treatment.

This study's limitations were its relatively low number of patients, its cross-sectional structure, and its evaluation of only a single inflammatory marker rather than the simultaneous evaluation of multiple markers.

\section{Conclusions}

This study showed that serum calprotectin levels are increased in AV patients, and this increase becomes more prevalent as the disease severity increases. Calprotectin can be a precise parameter in the evaluation of the severity of AV, but to clarify the contribution of calprotec- tin in the etiopathogenesis of AV and in clinical practice, future studies with larger series are needed.

\section{Conflict of interest}

The authors declare no conflict of interest.

\section{References}

1. Bhat YJ, Latief I, Hassan I. Update on etiopathogenesis and treatment of acne. Indian J Dermatol Venereol Leprol 2017; 83: 298-306.

2. Suh DH, Kwon HH. What's new in the physiopathology of acne? Br J Dermatol 2015; 172 Suppl 1: 13-9.

3. Stein Gold LF. What's new in acne and inflammation? J Drugs Dermatol 2013; 12: s67-9.

4. Jugeau S, Tenaud I, Knol AC, et al. Induction of Toll-like receptors by Propionibacterium acnes. Br J Dermatol 2005; 153: 1105-13.

5. Su Q, Grabowski M, Weindl G. Recognition of Propionibacterium acnes by human TLR2 heterodimers. Int I Med Microbiol 2017; 307: 108-12.

6. Gollnick HP. From new findings in acne pathogenesis to new approaches in treatment. J Eur Acad Dermatol Venereol 2015; 29 Suppl 5: 1-7.

7. Kristinsson J, Armbruster CH, Ugstad M, et al. Fecal cretion of calprotectin in colorectal cancer: relationship to tumor characteristics. Scand J Gastroenterol 2001; 36: 202-7.

8. El-Rifai W, Moskaluk CA, Abdrabbo MK, et al. Gastric cancers overexpress $\mathrm{S} 100$ calcium-binding proteins. Cancer Res 2002; 62: 6823-6.

9. Lee Y, Jang S, Min JK, et al. S100A8 and S100A9 are messengers in the crosstalk between epidermis and dermis modulating a psoriatic milieu in human skin. Biochem Biophys Res Commun 2012; 423: 647-53.

10. Dhas DBB, Bhat BV, Gane DB. Role of calprotectin in infection and inflammation. Curr Pediatr Res 2012; 16: 83-94.

11. De Ponti A, Wiechert L, Stojanovic A, et al. Chronic liver inflammation and hepatocellular carcinogenesis are independent of S100A9. Int J Cancer 2015; 136: 2458-63.

12. Gebhardt C, Németh J, Angel P, et al. S100A8 and S100A9 in inflammation and cancer. Biochem Pharmacol 2006; 72: 1622-31.

13. Bojko J. Measurement of blood calprotectin (MRP-8/MRP-14) levels in patients with juvenile idiopathic arthritis. Reumatologia 2017; 55: 10-4.

14. Berg-Hansen P, Vandvik B, Fagerhol M, et al. Calprotectin levels in the cerebrospinal fluid reflect disease activity in multiple sclerosis. J Neuroimmunol 2009; 216: 98-102.

15. Doshi A, Zaheer A, Stiller MJ. A comparation of current acne grading systems and proposal of a novel system. Int I Dermatol 1997; 36: 416-8.

16. Tanghetti EA. The role of inflammation in the pathology of acne. J Clin Aesthet Dermatol 2013; 6: 27-35.

17. Zhang M, Zhang X, Zhang J. Diagnostic value of fecal calprotectin in preterm infants with necrotizing enterocolitis. Clin Lab 2016; 62: 863-9.

18. Mariani A, Marsili M, Nozzi M, et al. Serum calprotectin: review of its usefulness and validity in paediatric rheumatic diseases. Clin Exp Rheumatol 2015; 33: 109-14.

19. Decembrino L, De Amici M, Pozzi M, et al. Serum calprotectin: a potential biomarker for neonatal sepsis. J Immunol Res 2015; 2015: 147973.

20. McNeill E, Hogg N. S100A9 has a protective role in inflammation-induced skin carcinogenesis. Int J Cancer 2014; 15: 798-808. 